\title{
Influência do tratamento preservativo com CCA-C na estabilidade dimensional da madeira de Eucalyptus
}

\author{
Effect of CCA-C preservative in the \\ dimensional stability of the eucalypt wood
}

\author{
Lorran Sousa Arantes', Paulo Ricardo Gherardi Hein'2, \\ José Reinaldo Moreira Silva ${ }^{3}$ e Vássia Carvalho Soares ${ }^{4}$
}

\begin{abstract}
Resumo
Objetivou-se avaliar em que medida o tratamento preservativo com CCA-C (Arseniato de Cobre Cromatado tipo ()), afeta a estabilidade dimensional da madeira de Eucalyptus tratada em autoclave. Foram utilizadas 78 amostras (39 não tratadas e 39 tratadas com CCA-C) de madeiras de Eucalyptus urophylla $\mathrm{x}$ E. grandis, na qual foram submetidas a diferentes condições de adsorção e dessorção de umidade, para posterior cálculo das contrações e inchamentos da madeira. Madeiras tratadas apresentaram maiores valores de inchamento radial ( $\alpha$ radial $=4,95 \%$ ) e tangencial ( $\alpha$ tangencial $=6,68 \%$ ) que as madeiras não tratadas ( $\alpha$ radial $=4,30 \%$ e $\alpha$ tangencial $=5,88 \%$ ) quando saíram de uma condição de $25{ }^{\circ} \mathrm{C}$ e $60 \%$ de umidade relativa do ar e foram submetidas a uma condição de saturação com $30{ }^{\circ} \mathrm{C} \mathrm{e} 80 \%$ de umidade relativa do ar. Quando saíram da condição anidra e foram submetidas à condição de $25{ }^{\circ} \mathrm{C}$ e $60 \%$ de umidade relativa do ar durante 30 dias houve efeito do tratamento preservativo com CCA-C apenas no inchamento tangencial. Contudo, quando as madeiras saturadas foram submetidas à condição anidra, a presença de sais metálicos não afetou os valores de contração. De modo geral, o tratamento preservativo com CCA-C não melhorou a estabilidade dimensional e o coeficiente de anisotropia das madeiras de Eucalyptus urophylla x E. grandis.
\end{abstract}

Palavras-chave: Contração. Inchamento. Coeficiente de anisotropia. Umidade.

\begin{abstract}
This study aimed to evaluate to what extent the treatment with the preservative CCA-C affects the dimensional stability of Eucalyptus wood treated on autoclaved. 78 samples were used (39 untreated and 39 treated with CCA-C) of Eucalyptus urophylla $\times$ E. grandis wood, which were submitted to different conditions of adsorption and desorption, for subsequent calculation of shrinkage and swelling of the wood. The treated wood pieces had a higher radial swelling values (a Radial $=4.95 \%$ ) and tangential (tangential $a=6.68 \%$ ) than the untreated wood $(a=4.30 \%$ radial and tangential $a=5.88 \%)$ when they came out of a condition of $25^{\circ} \mathrm{C}$ and $60 \%$ relative humidity, and were submitted to a saturation condition at $30^{\circ} \mathrm{C}$ and $80 \%$ relative humidity. When they left the dry condition and were subjected to the condition of $25^{\circ} \mathrm{C}$ and $60 \%$ relative humidity for 30 days, effect of preservative treatment with CCA-C only in tangential swelling were observed. However, when the saturated wood pieces were submitted to anhydrous condition, the presence of metal salts did not affect the shrinkage values. Generally, the preservative treatment with CCA-C has not improved dimensional stability, and the anisotropy coefficient of Eucalyptus urophylla $\times$ E. grandis wood.
\end{abstract}

Keywords: Shrinkage. Swelling. Anisotropy coefficient. Humidity.

\section{INTRODUÇÃO}

A madeira é um material higroscópico capaz de adsorver e perder água para a atmosfera causando alteração em suas dimensões, quando o teor de água da madeira encontra-se abaixo do ponto de saturação das fibras (PSF). A higroscopicidade da madeira se deve à natureza hidrofílica dos constituintes de sua parede celular, em que as hidroxilas presentes na celulose e hemiceluloses são polares

\footnotetext{
${ }^{1}$ Mestre em Ciência e Tecnologia da Madeira. UFLA - Universidade Federal de Lavras. Caixa postal 3037 -37200-000 Lavras. MG, Brasil. E-mail: lorran.ufla@gmail.com.

2Professor Adjunto no Departamento de Ciências Florestais. UFLA - Universidade Federal de Lavras. Caixa postal 3037 -37200-000 - Lavras. MG, Brasil. E-mail: paulo.hein@dcf.ufla.br.

3Professor Associado no Departamento de Ciências Florestais. UFLA - Universidade Federal de Lavras. Caixa postal 3037 -37200-000 - Lavras. MG, Brasil. E-mail: jreinaldo@dcf.ufla.br.

${ }^{4}$ Professora Doutora no Departamento de Ciências e Linguagens. IFMG - Instituto Federal de Minas Gerais. Rodovia Bambuí/Medeiros, Km 05 - Faz. Varginha, Bambuí - MG - 38900-000 E-mail: vassiasoares@gmail.com
}

Sci. For., Piracicaba, v. 45, n. 114, p. 285-293, jun. 2017 DOI: dx.doi.org/10.18671/scifor.v45n114.05 
e podem se ligar às moléculas de água (GALVÃO; JANKOWSKY, 1985). O estudo e o conhecimento das alterações dimensionais de madeiras de diferentes espécies, sobretudo nas de rápido crescimento, são essenciais para sua correta utilização industrial (TRIANOSKI et al., 2012).

A melhoria da estabilidade dimensional da madeira pode ser obtida pela redução da sua higroscopicidade, por meio da degradação térmica de seus constituintes mais hidrófilos (hemiceluloses), ruptura da celulose, criação de novas ligações químicas ou pela impregnação das paredes celulares da madeira com soluções salinas (KOLLMANN; CÔTÉ JUNIOR, 1968).

Para amenizar a instabilidade dimensional da madeira, Kollmann (1959) investigou o emprego de soluções alcalinas e ácidas e observou que o inchamento da madeira foi menor que aquele resultante da adsorção de água pura. Stamm (1977) relatou que algumas soluções de sais e açúcares concentrados podem ser utilizadas para reduzir o inchamento e contração da madeira, tais como: soluções de cloreto de manganês, de sódio, de bário, de magnésio e de lítio, além de soluções de sacarose, glucose e frutose. Segundo Kollmann e Côté Junior (1968), a estabilidade da madeira tratada com sais ocorre por causa da reação destes compostos com os constituintes da parede celular, formando um complexo, que impede a madeira de adsorver água, uma vez que sua estrutura já se encontra saturada com os sais.

Paes et al. (2015) constataram que soluções salinas de cloreto de lítio e de sulfato de cobre II promoveram a melhoria da estabilidade dimensional da madeira de Corymbia torelliana. Os mesmos autores encontraram melhorias da estabilidade dimensional de madeiras de Eucalyptus cloeziana quando estas foram tratadas com soluções de cloreto de sódio e sulfato de cobre II. Paes et al. (2013) verificaram que a impregnação de soluções salinas (cloreto de lítio, cloreto de sódio, sulfato de alumínio, sulfato de magnésio e sulfato de zinco) na madeira de Paulownia tomentosa não melhoraram a sua estabilidade dimensional. Xie et al. (2011) observaram melhoria da estabilidade dimensional de madeiras de Pinus sylvestris L. tratadas com solução de glutaraldeído e cloreto de magnésio.

Takeshita (2016) após submeter madeiras de Cedrinho (Erisma uncinatum), Tachi preto (Tachigali myrmecophyla) e Itaúba amarela (Mezilaurus itauba) a tratamentos estabilizadores em autoclave a 90 ${ }^{\circ} \mathrm{C}(24$ e $96 \mathrm{~h})$ e $120{ }^{\circ} \mathrm{C}$ ( 2 e 8h), e posterior condicionamento higroscópico em soluções salinas de cloreto de magnésio, brometo de sódio e sulfato de potássio, não verificou redução significativa da higroscopicidade e da variação dimensional para tais madeiras.

O Arseniato de Cobre Cromatado (CCA) é um sal preservativo, amplamente utilizado na preservação de madeiras, protegendo-a da podridão por fungos e da deterioração por insetos e agentes marinhos. O produto é uma combinação básica de Cu (cobre), Cr (cromo) e As (arsênio). O CCA apresenta ampla utilização em todo mundo, sendo que no Brasil, trata-se de um produto de uso exclusivamente industrial, devendo ser, portanto utilizado apenas por usinas de preservação de madeiras devidamente registradas no Instituto Brasileiro do Meio Ambiente e dos Recursos Renováveis (IBAMA). Apesar de sua ampla utilização, ainda são poucas as informações na literatura a respeito da influência do CCA na estabilidade dimensional da madeira, sobretudo na madeira de Eucalyptus.

Considerando que a madeira de Eucalyptus é amplamente empregada das mais variadas formas e que este gênero apresenta elevada anisotropia e instabilidade dimensional, é importante conhecer melhor o comportamento da madeira de Eucalyptus sob determinadas condições de uso, visando melhoria de sua qualidade em termos de estabilidade dimensional.

Por isso, este trabalho teve como objetivo verificar em que medida o tratamento preservativo com CCA-C (Arseniato de Cobre Cromatado tipo C) afeta a estabilidade dimensional da madeira de Eucalyptus tratada em autoclave.

\section{MATERIAL E MÉTODOS}

\section{Material vegetal e amostragem}

Neste trabalho, 14 moirões de madeira de Eucalyptus urophylla $\mathrm{x}$ E. grandis, com diâmetro variando de 9 a $12 \mathrm{~cm}$ e comprimento de 1,60 m, fornecidos pela empresa Esteio S.A. localizada em São João Del Rei/MG, provenientes de um plantio clonal de 5 anos foram investigados. Os moirões avaliados neste estudo foram selecionados em função da umidade, pré-determinada com auxílio de um medidor elétrico. Moirões com umidade entre 15 e 40\% de acordo com o indicador foram selecionados e avaliados. 
Após a seleção dos moirões, discos de $5 \mathrm{~cm}$ foram retirados das extremidades dos moirões, e descartados por apresentar rachaduras e umidade relativamente mais baixa. Após esta operação discos de $5 \mathrm{~cm}$ de espessura foram retirados de uma das extremidades e da parte central dos moirões, e identificados para determinação da umidade e estabilidade dimensional da madeira sem tratamento conforme norma NBR 7190 (ABNT, 1997).

Os toretes resultantes desses processamentos foram identificados e submetidos ao tratamento preservativo em autoclave na empresa Esteio S.A. para impregnação de CCA tipo C, com concentração de 1,7\%, conforme procedimento industrial adotado pela empresa. Após o tratamento, discos de $5 \mathrm{~cm}$ de espessura de uma das extremidades dos toretes tratados foram retirados para análises de retenção e para determinação da estabilidade dimensional da madeira tratada.

\section{Determinação da estabilidade dimensional da madeira}

Corpos de prova com dimensões nominais de $20 \mathrm{~mm}$ x $20 \mathrm{~mm}$ x $50 \mathrm{~mm}$ ( $\mathrm{R}$ x T x L) foram retirados da região tratável (alburno) dos discos para a determinação da variação dimensional da madeira tratada e não tratada. Foram utilizados 78 corpos de prova (39 tratados e 39 não tratados). Esses corpos de prova foram submetidos a diversas condições de umidade relativa do ar com o objetivo de induzir variação na massa e nas dimensões dos corpos de prova em função da adsorção ou dessorção de umidade. Em cada condição, a massa e a dimensão nas superfícies longitudinal-radial e longitudinal-tangencial dos corpos de prova tratados e não tratados foram determinadas para cálculo da umidade e das contrações e inchamentos nas diferentes situações. Os locais das medições foram anteriormente marcados com caneta esferográfica para que as medições fossem realizadas nos mesmos locais. As condições nas quais as amostras de madeira tratada e não tratada foram submetidas são apresentadas abaixo:

\section{Condição 1 (ambiente)}

Primeiramente os corpos de provas (tratados e não tratados) foram mantidos em câmara climatizada (Temp: $25^{\circ} \mathrm{C}$ e UR: 60\%), de modo a entrar em equilíbrio higroscópico e estabilizar a umidade de equilíbrio da madeira. Após a estabilização das massas, os corpos de prova foram pesados em balança de precisão e suas dimensões foram determinadas na superfície longitudinal-radial e longitudinal-tangencial com auxílio de paquímetro digital.

\section{Condição 2 (saturação)}

Os corpos de prova foram submetidos à condição de saturação em casa de vegetação com sistema de irrigação e ventilação. A Temperatura média foi de $30{ }^{\circ} \mathrm{C}$ e a UR média foi de $80 \%$. Os corpos de prova permaneceram nessas condições de alta umidade durante 5 dias, até que a massa se estabilizou. Com base nas massas e nas dimensões, o inchamento dos corpos de prova foi calculado no sentido radial e tangencial.

\section{Condição 3 (anidra)}

Os corpos de prova foram submetidos à secagem ao ar livre durante 20 dias e em seguida foram colocados em estufa a $103^{\circ} \mathrm{C}$ até atingirem massa constante. As dimensões e a massa seca das amostras foram determinadas para cálculo das contrações radiais e tangenciais e da umidade das demais condições.

\section{Condição 4 (ambiente)}

Os corpos de prova retornaram à câmara climatizada da condição 1 (Temp: $25^{\circ} \mathrm{C}$ e UR: 60\%) durante 30 dias para retomada da condição de equilíbrio com o ambiente. Esse período foi suficiente para que todas as amostras atingissem massa constante. Após a estabilização das massas, os corpos de prova foram pesados e as dimensões remedidas de modo a se calcular o inchamento dos corpos de provas de madeira tratada e não tratada.

A umidade, as contrações e inchamentos das amostras de madeira tratada e não tratada foram calculadas de acordo com a norma NBR 7190 (ABNT, 1997). O coeficiente anisotrópico, para a contração e o inchamento, foi obtido pelo quociente entre as dimensões tangenciais e as radiais. 


\section{Análise de retenção}

Amostras de madeira para análise de retenção foram retiradas dos discos em regiões próximas de onde foram cortadas as amostras dos testes da estabilidade dimensional (área tratável). As amostras foram processadas em moinho martelo e classificadas em peneira de granulometria de 40 mesh, onde se obteve 14 amostras compostas provenientes dos moirões.

As amostras foram submetidas à digestão acelerada, por meio de solução nitroperclórica. A quantificação dos elementos cobre, cromo e arsênio presentes nas amostras foi realizada por espectrofotometria de absorção atômica. Os cálculos de retenção foram efetuados de acordo com a equação 1, apresentada por Paes (1991).

$$
R=\left(F \times \operatorname{Lx} F d \times 10^{-3}\right) / V
$$

em que: $\mathrm{R}$ = retenção do elemento na madeira $\left(\mathrm{kg} \mathrm{I} . \mathrm{A} . / \mathrm{m}^{3}\right) ; \mathrm{F}=$ fator estequiométrico empregado para transformação dos elementos químicos para óxidos (cobre = 1,2518; cromo = 1,9230; arsênio = 3,0680); L = leitura obtida do espectrofotômetro $(\mathrm{mg} / \mathrm{L}) ; \mathrm{Fd}=$ fator de diluição necessário para as leituras no espectrofotômetro; $\mathrm{V}=$ volume das amostras utilizadas nas análises $\left(\mathrm{cm}^{3}\right)$.

Os cálculos foram feitos para cada elemento químico individualmente, sendo que a retenção total corresponde à somatória das retenções individuais.

\section{Análise estatística}

Para avaliar o efeito do CCA-C na estabilidade dimensional da madeira e na umidade de equilíbrio em cada condição, foi feita a comparação entre médias das madeiras tratadas e não tratadas, por meio do teste $t$ a $5 \%$ de significância. Avaliou-se também a influência da retenção na estabilidade dimensional e na umidade das madeiras tratadas com CCA-C por meio da correlação de Pearson. As análises estatísticas foram realizadas por meio do programa estatístico SPSS Statistics v.19.

\section{RESULTADOS E DISCUSSÃO}

\section{Umidade de equilíbrio da madeira}

Os valores de umidade dos corpos de prova tratados e não tratados nas condições ambiente (Condição 1 e 4) e de saturação (Condição 2) no ensaio de estabilidade dimensional se encontram na Tabela 1. Verifica-se que a umidade dos corpos de provas tratados e não tratados com CCA-C não diferiram estatisticamente quando submetidos à saturação (Condição 2). Porém, nas condições ambientais 1 e 4, os corpos de prova tratados com CCA-C apresentaram uma maior umidade em relação aos não tratados. Na condição 3, a umidade das amostras foi de zero por cento.

Tabela 1. Valores de umidade de Eucalyptus urophylla x E. grandis tratados e não tratados com CCA-C no ensaio de estabilidade dimensional.

Table 1. Humidity values of Eucalyptus urophylla x E. grandis treated and not treated with CCA-C in the dimensional stability test.

\begin{tabular}{lcc}
\hline \multirow{2}{*}{ Condição } & \multicolumn{2}{c}{ Umidade (\%) } \\
\cline { 2 - 3 } & Tratado & Não tratado \\
\hline Condição 1 (ambiente) & $12,51 \mathrm{a}(4,57)$ & $12,22 \mathrm{~b}(5,13)$ \\
Condição 2 (saturação) & $60,41 \mathrm{a}(19,88)$ & $62,59 \mathrm{a}(16,29)$ \\
Condição 3 (anidra) & 0 & 0 \\
Condição 4 (ambiente) & $11,59 \mathrm{a}(2,25)$ & $11,31 \mathrm{~b}(3,12)$ \\
\hline
\end{tabular}

Médias seguidas de mesma letra na horizontal não diferem estatisticamente pelo teste $t(p>0,05)$. Valores entre parênteses correspondem ao coeficiente de variação, em \%.

A Figura 1 apresenta o diagrama de extremos e quartis da umidade de equilíbrio das madeiras tratadas e não tratadas submetidas às condições 1 (ambiente), 2 (saturação) e 4 (ambiente). As condições ambientais 1 e 4 são idênticas, mas na situação 1 a madeira estava numa condição saturada e dessorveu água até atingir o equilíbrio ao passo que na situação 4 a madeira estava numa condição anidra e adsorveu água até atingir o equilíbrio. 

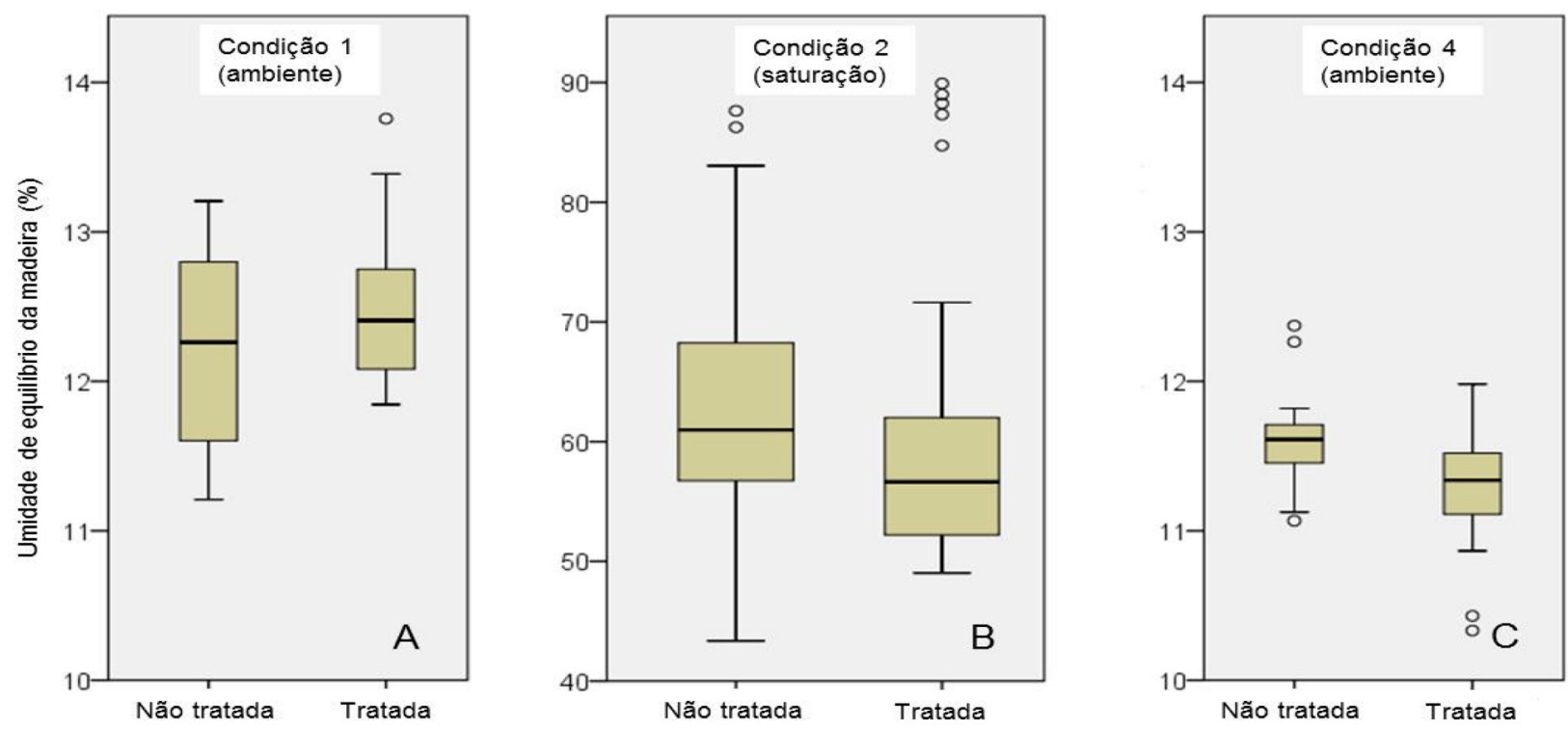

Figura 1. Umidade de equilíbrio da madeira de Eucalyptus urophylla x E. grandis tratadas e não tratadas submetidas a diferentes situações.

Figure 1. Moisture balance of Eucalyptus urophylla x E. grandis treated and untreated under different situations.

\section{Retratibilidade da madeira}

Os valores médios da retratibilidade e o coeficiente de anisotropia (CA) de madeiras tratadas e não tratadas com CCA-C em diferentes situações são apresentados na Tabela 2. Os corpos de prova tratados com CCA-C apresentaram maiores valores de inchamento radial e tangencial após estabilização na condição de saturação (Condição 1 para Condição 2). O inchamento radial das madeiras tratadas e não tratadas com CCA-C, após 30 dias em câmara climatizada, foi semelhante para os dois tratamentos; quanto ao inchamento tangencial, este foi maior para madeiras tratadas com CCA-C (Condição 3 para Condição 4).

Tabela 2. Contrações e inchamentos nos sentidos radial, tangencial e coeficiente de anisotropia (CA) de madeiras de Eucalyptus urophylla x E. grandis tratadas e não tratadas com CCA-C em diferentes situações.

Table 2. Shrinkage and swelling in the radial and tangential sense and anisotropy coefficient (CA) of Eucalyptus urophylla $x$ E. grandis woods treated and not treated with CCA-C in different situations.

\begin{tabular}{llcc}
\hline \multirow{2}{*}{ Situação } & \multirow{2}{*}{ Estabilidade dimensional } & \multicolumn{2}{c}{ Tratamentos } \\
\cline { 3 - 4 } & & Tratado & Não tratado \\
\hline \multirow{3}{*}{ Inchamento (Cond 1 para Cond 2) } & $\alpha$ radial & $4,95 \mathrm{a}(29,35)$ & $4,30 \mathrm{~b}(31,28)$ \\
& $\alpha$ tangencial & $6,68 \mathrm{a}(25,30)$ & $5,88 \mathrm{~b}(21,37)$ \\
& $\mathrm{CA}$ (inchamento) & $1,46 \mathrm{a}(39,59)$ & $1,52 \mathrm{a}(48,16)$ \\
\hline \multirow{3}{*}{ Contração (Cond 2 para Cond 3) } & $\beta$ radial & $6,17 \mathrm{a}(22,35)$ & $5,69 \mathrm{a}(21,46)$ \\
& $\beta$ tangencial & $7,93 \mathrm{a}(18,56)$ & $7,34 \mathrm{a}(24,09)$ \\
& $\mathrm{CA}$ (contração) & $1,36 \mathrm{a}(38,08)$ & $1,33 \mathrm{a}(26,47)$ \\
\hline \multirow{3}{*}{ Inchamento (Cond 3 para Cond 4) } & $\alpha$ radial & $2,02 \mathrm{a}(42,32)$ & $1,93 \mathrm{a}(34,30)$ \\
& $\alpha$ tangencial & $2,90 \mathrm{a}(26,21)$ & $2,49 \mathrm{~b}(26,10)$ \\
& CA (inchamento) & $1,72 \mathrm{a}(58,59)$ & $1,49 \mathrm{a}(59,64)$ \\
\hline
\end{tabular}

Médias seguidas de mesma letra na horizontal não diferem estatisticamente pelo teste $t(p>0,05)$. Valores entre parênteses correspondem ao coeficiente de variação, em \%.

Esses resultados divergem com as afirmações de Kollmann e Côté Junior (1968) de que a impregnação da madeira com sais ocasiona um menor inchamento da madeira devido à retenção de umidade pelos sais, o que minimiza o fenômeno de adsorção de moléculas de água nos espaços submicroscópicos das micelas.

Paes et al. (2015) avaliaram a influência de soluções salinas na estabilidade dimensional das madeiras de Corymbia torelliana e Eucalyptus cloeziana. Estes autores verificaram para madeira de Corymbia torelliana menores valores de inchamento radial e tangencial para as madeiras impregnadas com solução de cloreto de lítio. Para madeira de Eucalyptus cloeziana, os autores encontraram um menor inchamento radial para as amostras impregnadas com cloreto de lítio, e para o inchamento tangencial menores valores foram encontrados para aquelas impregnadas com soluções de cloreto de 
lítio, carbonato de sódio, sulfato de magnésio, sulfato de zinco e sulfato de cobre II em relação às amostras imersas em água destilada (controle).

A Figura 2 apresenta a variação dos níveis de inchamento das madeiras tratadas e não tratadas submetidas a diferentes situações. O diagrama de caixa da Figura 2A apresenta à mediana e os quartis dos níveis de inchamento da madeira que estava na câmera climatizada e foi submetida a ambiente de alta saturação enquanto a Figura $2 \mathrm{~B}$ apresenta a variação do inchamento das mesmas madeiras da condição anidra até atingirem massa constante quando colocadas novamente em câmara climática.

Em relação à contração radial e tangencial das madeiras, não houve diferença significativa entre as amostras tratadas e não tratadas com CCA-C (Tabela 2). Para madeiras de Corymbia torelliana Paes et al. (2015) também não encontraram diferenças significativas entre as contrações radial e tangencial das amostras de madeiras impregnadas com as diversas soluções salinas e aquelas imersas em água destilada, exceto para as madeiras impregnadas com carbonato de sódio, na qual apresentaram uma maior contração tangencial em relação ao controle. Os mesmos autores encontraram para as madeiras de Eucalyptus cloeziana resultados semelhantes aos encontrados para as madeiras de Corymbia torelliana, onde as amostras de madeiras impregnadas com as diversas soluções salinas apresentaram contrações radiais e tangenciais semelhantes ao controle, exceção foi encontrada para as amostras impregnadas com cloreto de sódio, que apresentaram uma menor contração tangencial em relação ao controle.
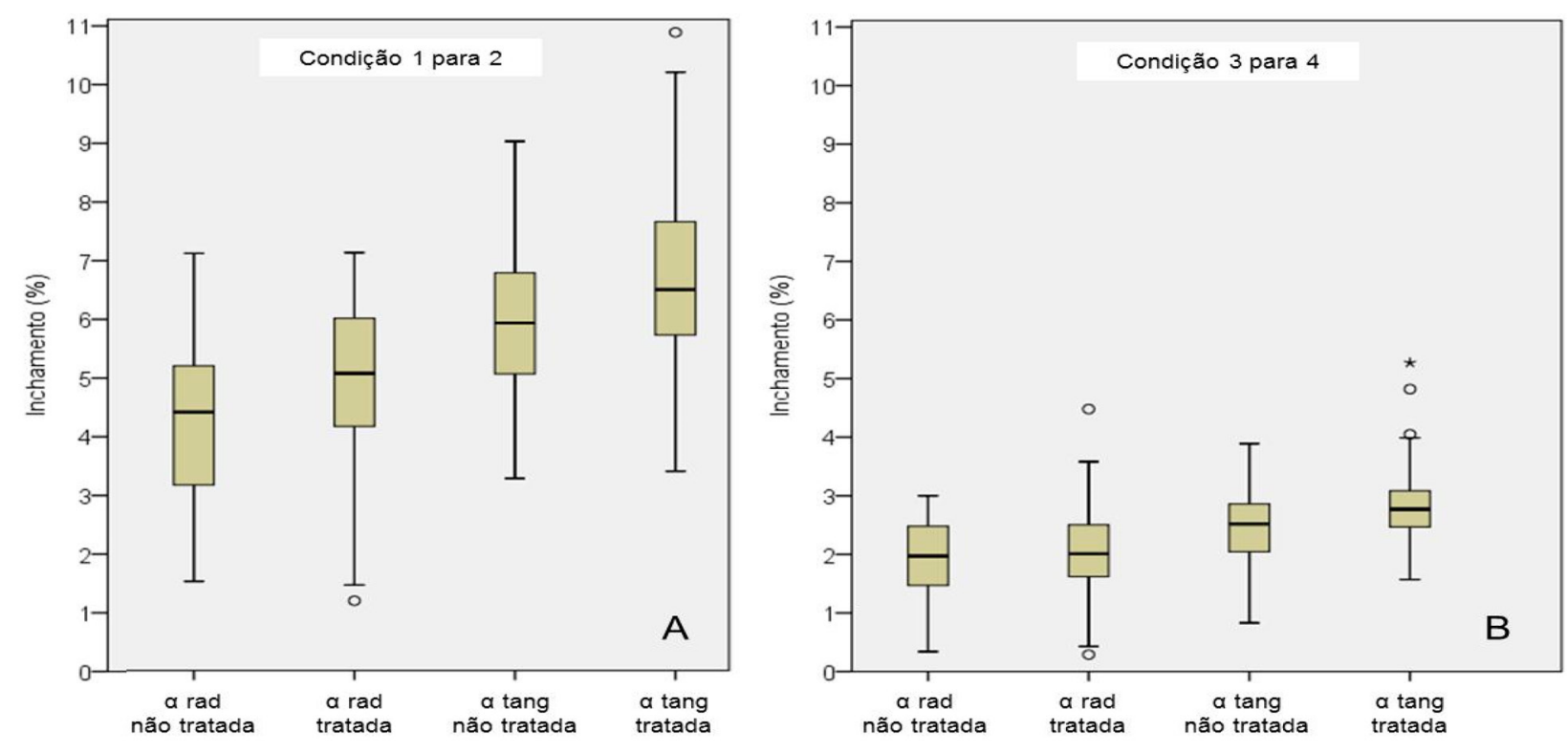

Figura 2. Inchamento radial e tangencial das madeiras de Eucalyptus urophylla $x$ E. grandis tratadas e não tratadas submetidas em duas situações.

Figure 2. Radial and tangential swelling of wood Eucalyptus urophylla x E. grandis treated and untreated submitted in two situations.

Os resultados de contração radial e tangencial encontrados neste trabalho se assemelham aos encontrados por Paes et al. (2015). Uma forma de reduzir a contração ou o inchamento da madeira é através do preenchimento dos espaços vazios existentes na estrutura da madeira com produtos químicos capazes de reagir com os constituintes da parede celular da madeira (COSTA et al., 2001). O CCA-C é um produto que durante as suas reações de fixação na madeira pode degradar a hemicelulose e formar compostos com a celulose, o que pode levar há uma diminuição da higroscopicidade da madeira, e consequentemente uma redução da sua instabilidade dimensional.

Pode-se reduzir as variações dimensionais da madeira através da reação de componentes químicos com as hidroxilas presentes na celulose, de modo a não deixar estas disponíveis para interagir com a água (GOMES et al., 2006). Segundo Lepage (1986), após a fixação do CCA na madeira, uma grande quantidade de cobre fica retida na camada S1 da parede celular, que se adere à lignina e também a celulose através das ligações $\mathrm{OH}$.

Com relação ao coeficiente anisotrópico, este não diferiu estatisticamente entre as madeiras tratadas e não tratadas com CCA-C para os inchamentos e também para contração (Tabela 2). 
Observando os valores de coeficiente anisotrópico, verifica-se que as madeiras apresentaram boa estabilidade dimensional, devido aos baixos valores de CA encontrados para as madeiras tratadas e às não tratadas. Segundo Klitzke (2007) madeiras com coeficiente de anisotropia inferior a 1,5 são consideradas madeiras estáveis.

Através dos resultados obtidos, observa-se que o tratamento preservativo com CCA-C não melhorou a estabilidade dimensional da madeira de Eucalyptus urophylla x E. grandis.

\section{Correlações entre a retenção de CCA-C e as propriedades da madeira tratada}

Os valores médios de retenção de CCA-C e de umidade da madeira no momento do tratamento preservativo de moirões de Eucalyptus urophylla $\mathrm{x}$ E. grandis se encontram na Tabela 3. De acordo com a norma NBR 9480 (ABNT, 2009), a retenção mínima indicada para moirões, uso em contato com o solo, é de $6,5 \mathrm{~kg}$ I.A./ $\mathrm{m}^{3}$, porém, a retenção média dos moirões utilizados neste trabalho foi menor do que o exigido pela norma, na qual atingiram uma retenção de $6,23 \mathrm{~kg}$ I.A./ $/ \mathrm{m}^{3}$.

Tabela 3. Retenção de CCA-C em kg I.A./ $\mathrm{m}^{3}$ e umidade (\%) da madeira antes do tratamento preservativo de moirões de Eucalyptus urophylla x E. grandis.

Table 3. Retention of CCA-C in kg E.M. $/ \mathrm{m}^{3}$ and humidity (\%) of the wood before preservative treatment of fence posts from Eucalyptus urophylla x E. grandis.

\begin{tabular}{ccc|ccc}
\hline Moirão & Retenção & Umidade & Moirão & Retenção & Umidade \\
\hline 1 & 5,61 & 32,65 & 9 & 6,14 & 26,62 \\
2 & 5,68 & 30,65 & 10 & 5,87 & 38,37 \\
3 & 6,84 & 19,55 & 11 & 6,29 & 30,24 \\
4 & 5,26 & 32,09 & 12 & 7,58 & 25,72 \\
5 & 6,25 & 26,06 & 13 & 6,36 & 28,46 \\
6 & 6,95 & 22,02 & 14 & 5,98 & 34,14 \\
7 & 6,07 & 33,90 & Média & 6,23 & 28,84 \\
8 & 6,38 & 23,32 & CV (\%) & 9,55 & 18,27 \\
\hline
\end{tabular}

$\overline{C V}=$ Coeficiente de variação.

A umidade da madeira afeta a retenção de produtos preservativos, uma vez que a umidade da madeira acima do ponto de saturação das fibras (PSF) pode impedir que a madeira atinja a retenção de preservativo desejada quando esta é tratada em autoclave (LEPAGE, 1986). Observa-se pela Tabela 3, que a umidade média dos moirões foi de $28,84 \%$, atendendo as recomendações para o tratamento preservativo em autoclave.

Tabela 4. Coeficientes de correlação entre a retenção de CCA-C e as propriedades da madeira.

Table 4. Coefficients of correlation between the retention of CCA-C and the properties of the wood.

\begin{tabular}{lc}
\hline Retenção de CCA-C & (r) \\
\hline Versus & $-0,30$ \\
U2 & 0,31 \\
U4 & $\mathrm{ns}$ \\
Inchamento radial (Cond 1 para 2) & $\mathrm{ns}$ \\
Inchamento tangencial (Cond 1 para 2) & $-0,22$ \\
Contração radial (Cond 2 para 3) & $\mathrm{ns}$ \\
Contração tangencial (Cond 2 para 3) & $\mathrm{ns}$ \\
Inchamento radial (Cond 3 para 4) & $\mathrm{ns}$ \\
Inchamento tangencial (Cond 3 para 4) & $-0,32$ \\
\hline
\end{tabular}

ns = não significativo pela correlação de Pearson a 5\% de significância; UI = umidade na condição ambiente (câmara climatizada); U2 = umidade na condição de saturação; $U 4$ = umidade após 30 dias em câmara climatizada.

Com os valores de retenção de CCA-C foi possível fazer o estudo das correlações entre estes e as propriedades da madeira tratada (Tabela 4). A retenção de CCA-C influenciou de forma fraca nos valores de umidade U1 (câmara climatizada) e U2 (condição saturada). A retenção influenciou negativamente de forma fraca nos valores de inchamento tangencial, e não afetou o inchamento radial nem as contrações radial e tangencial da madeira de Eucalyptus urophylla $\mathrm{x}$ E. grandis tratadas, devido a falta de correlações entre eles. 
Arantes et al. - Influência do tratamento preservativo com

CCA-C na estabilidade dimensional da madeira de Eucalyptus

\section{CONCLUSÕES}

Madeiras tratadas apresentaram uma umidade de equilíbrio significativamente maior $(\mathrm{U}=$ $12,51 \%$ ) quando submetidas a uma condição de $25{ }^{\circ} \mathrm{C}$ e $60 \%$ de umidade relativa do ar do que madeiras não tratadas $(\mathrm{U}=12,22 \%)$. Por outro lado, quando as madeiras adsorvem água do estado anidro até a condição de $25{ }^{\circ} \mathrm{C}$ e $60 \%$ de umidade relativa do ar, as madeiras tratadas apresentaram umidade de equilíbrio significativamente superior $(U=11,59 \%)$ em relação à madeira não tratada $(U=11,31 \%)$. Entretanto, não houve diferença significativa na umidade de equilíbrio quando as madeiras tratadas e não tratadas foram submetidas à saturação em uma condição de alta umidade relativa do ar.

Madeiras tratadas apresentaram maiores valores de inchamento radial ( $\alpha$ radial $=4,95 \%)$ e tangencial ( $\alpha$ tangencial $=6,68 \%$ ) que as madeiras não tratadas $(\alpha$ radial $=4,30 \%$ e $\alpha$ tangencial $=$ $5,88 \%$ ) quando saíram de uma condição de $25{ }^{\circ} \mathrm{C}$ e $60 \%$ de umidade relativa do ar e foram submetidas a uma condição de saturação com $30{ }^{\circ} \mathrm{C}$ e $80 \%$ de umidade relativa do ar. Quando saíram da condição anidra e foram submetidas à condição de $25{ }^{\circ} \mathrm{C}$ e $60 \%$ de umidade relativa do ar durante 30 dias houve efeito do tratamento preservativo com CCA-C apenas no inchamento tangencial. Contudo, quando as madeiras saturadas foram submetidas à condição anidra, a presença de sais metálicos não afetou os valores de contração.

De modo geral, o tratamento preservativo com CCA-C não melhorou a estabilidade dimensional e o coeficiente de anisotropia das madeiras de Eucalyptus urophylla x E. grandis.

\section{REFERÊNCIAS BIBLIOGRÁFICAS}

ABNT - ASSOCIAÇÃO BRASILEIRA DE NORMAS TÉCNICAS. NBR 7190: projeto de estruturas de madeira. Rio de Janeiro, 1997. 107 p.

ABNT - ASSOCIAÇÃO BRASILEIRA DE NORMAS TÉCNICAS - ABNT. NBR 9480: peças roliças preservadas de eucalipto para construções rurais. Rio de Janeiro: ABNT, 2009. 12 p.

COSTA, A. F.; VALE, A. T.; GONÇALEZ, J. C. Eficiência de um resíduo de origem petrolífera sobre a estabilidade dimensional da madeira de Pinus sp. (pinus) e Mimosa scabrella Bentham (bracatinga). Ciência Florestal, Santa Maria, v. 11, n. 2, p. 59-70, 2001.

GALVÃO, A. P. M.; JANKOWSKY, I. P. Secagem racional da madeira. São Paulo: Nobel, 1985. 112 p.

GOMES, D. F. F.; SILVA, J. R. M.; BIANCHI, M. L.; TRUGILHO, P. F. Avaliação da estabilidade dimensional da madeira acetilada de Eucalyptus grandis Hill ex. Maiden. Scientia Forestalis, Piracicaba, n. 70, p. 125-130, 2006.

KLITZKE, R. J. Secagem da madeira. In: OLIVEIRA, J. T. S.; FIEDLER, N. C.; NOGUEIRA, N. (Orgs.). Tecnologias aplicadas ao setor madeireiro. Visconde do Rio Branco: Suprema Gráfica e Editora, 2007. v. 1, p. 271366.

KOLLMANN, F. E. P. Tecnologia de la madera y sus aplicaciones. Madrid: Gráficas Reunidas S.A., 1959. 675 p.

KOLLMANN, F. E. P.; CÔTÉ JUNIOR, W. A. Principles of wood science and technology. New York: SpringerVerlarg, 1968. 592 p.

LEPAGE, E. S. Manual de preservação de madeiras. São Paulo: IPT, 1986.

PAES, J. B. Viabilidade do tratamento preservativo de moirões de bracatinga (Mimosa scabrella Benth.), por meio de métodos simples e comparações de sua tratabilidade com a do Eucalyptus viminalis Lab. Dissertação (Mestrado em Ciência Florestal) - Universidade Federal do Paraná, Curitiba, 1991. 140 p. 
PAES, J. B.; LOIOLA, P. L.; EUFLOSINO, A. E. R.; SALVADOR, F. M.; VITÓRIA, J. B. Efeito de soluções salinas na estabilidade dimensional da madeira de Kiri (Paulownia tomentosa). Revista Científica Eletrônica de Engenharia Florestal, Garça, v. 21, n. 1, p. 72-84. 2013.

PAES, J. B.; LOIOLA, P. L.; OLIVEIRA, J. T. S.; BRAZ, R. L.; KLITZKE, R. J. Efeito de soluções salinas na estabilidade dimensional das madeiras de Corymbia torelliana e Eucalyptus cloeziana. Scientia Forestalis, Piracicaba, v. 43, n. 106, p. 271-281, 2015.

STAMM, A. J. Dimensional changes of wood and their control. In: GOLDSTEIN, I.S. (Ed.) Wood technology: chemical aspects. Washington: ACS Publication, 1977. p.115-140.

TAKESHITA, S. Aplicação de tratamentos estabilizadores pós-secagem convencional visando a redução do potencial higroscópico da madeira. 2016. 146 p.Tese (Doutorado em Ciências) - Universidade de São Paulo, Escola Superior de Agricultura "Luiz de Queiroz", Piracicaba, 2016.

TRIANOSKI, R.; MATOS, J. L. M.; IWAKIRI, S.; PRATA, J. G. Avaliação da estabilidade dimensional de espécies de pinus tropicais. Floresta e Ambiente, Seropédica, v. 20, n. 3, p. 398-406. 2012.

XIE, Y.; HILL, C. A. S.; XIAO, Z.; MAI, C.; MILITZ, H. Dynamic water vapour sorption properties of wood treated with glutaraldehyde. Wood Science and Technology, Berlin, v. 48, n. 1, p. 49-61. 2011.

Recebido em 08/04/2016

Aceito para publicação em 10/01/2017

Sci. For., Piracicaba, v. 45, n. 114, p. 285-293, jun. 2017 DOI: dx.doi.org/10.18671/scifor.v45n114.05 
